\title{
THE CLASSIFICATION OF REGULATORY SANDBOXES (EXPERIMENTAL LEGAL REGIMES): RUSSIAN AND FOREIGN EXPERIENCE ${ }^{1}$
}

\author{
Vladislav O. Makarov \\ Volgograd State University, Volgograd, Russian Federation
}

Introduction: the institution of regulatory sandboxes, which is a financial and legal tool aimed at testing new technological solutions, which is not subject to restrictions of the current legal regulation, has been developed in almost all countries of the world, including the Russian Federation. At the same time, a theoretical and comparative legal study of a rapidly developing institution is required. In the paper, we study the types of regulatory sandboxes that are distinguished in world practice, as well as propose other classifications of experimental legal regimes. Purpose: based on Russian and foreign experience, to identify approaches to the regulation of digital innovations and the classification of experimental legal regimes (regulatory sandboxes) using various criteria. The research methodology is based on the application of methods of formal logic, system, and structural analysis, as well as the comparative law method, the methods of legal experiment, legal forecasting, and interpretation of legal norms. As a result of the conducted research, four main approaches to the regulation of digital innovations are described and adapted, as well as the classifications of regulatory sandboxes (experimental legal regimes) are identified. Conclusions: the main approaches to the regulation of innovations in international practice are observational, approbation, stimulating, and reformation. In turn, it is possible to classify regulatory sandboxes based on Russian and foreign experience by goals, by the territory of operation, by the subject of expertise, by the duration of testing, by the type of solutions promoted, by consumer protection measures, by the regulatory body, as well as by the scope of technology application.

Key words: experimental legal regimes, regulatory sandboxes, fintech, digital economy, digital innovations, legal experiment, legal regime, classification.

Citation. Makarov V.O. The Classification of Regulatory Sandboxes (Experimental Legal Regimes): Russian and Foreign Experience. Legal Concept = Pravovaya paradigma, 2021, vol. 20, no. 3, pp. 35-41. (in Russian). DOI: https://doi.org/10.15688/lc.jvolsu.2021.3.6

УДК 340.5

Дата поступления статьи: 10.05.2021

ББК 67.0

Дата принятия статьи: 14.06.2021

\section{КЛАССИФИКАЦИЯ РЕГУЛЯТИВНЫХ ПЕСОЧНИЦ (ЭКСПЕРИМЕНТАЛЬНЫХ ПРАВОВЫХ РЕЖИМОВ): РОССИЙСКИЙ И ЗАРУБЕЖНЫЙ ОПЫТ ${ }^{1}$}

\author{
Владислав Олегович Макаров \\ Волгоградский государственный университет, г. Волгоград, Российская Федерация
}

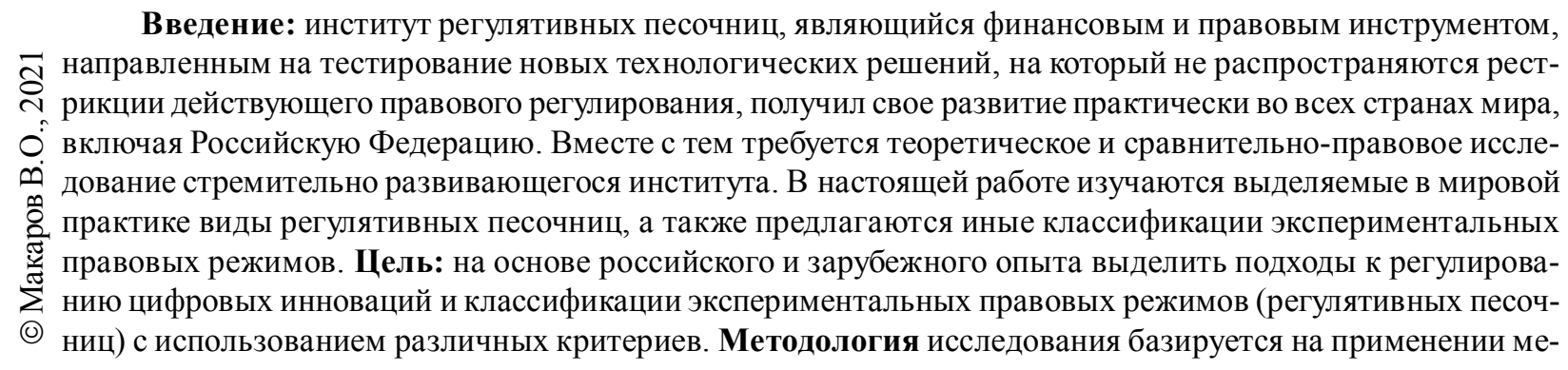


тодов формальной логики, системного и структурного анализа, а также сравнительно-правового метода, методов правового эксперимента, правового прогнозирования и толкования правовых норм. В результате проведенного исследования описаны и адаптированы четыре основных подхода к регулированию цифровых инноваций, а также выделены классификации регулятивных песочниц (экспериментальных правовых режимов). Выводы: основными подходами к регулированию инноваций в международной практике являются наблюдательный, апробационный, стимулирующий и реформационный. В свою очередь, классифицировать регулятивные песочницы на основе российского и зарубежного опыта можно по целям, по территории функционирования, по субъекту проведения экспертизы, по продолжительности тестирования, по виду продвигаемых решений, по мерам защиты потребителя, по регулирующему органу, а также по сфере применения технологий.

Ключевые слова: экспериментальные правовые режимы, регулятивные песочницы, финтех, цифровая экономика, цифровые инновации, правовой эксперимент, правовой режим, классификация.

Цитирование. Макаров В. О. Классификация регулятивных песочниц (экспериментальных правовых режимов): российский и зарубежный опыт // Legal Concept = Правовая парадигма. - 2021. - Т. 20, № 3. - C. 35-41.DOI: https://doi.org/10.15688/lc.jvolsu.2021.3.6

\section{Введение}

За последние 5 лет институт регулятивных песочниц, являющийся финансовым и правовым инструментом, направленным на тестирование новых технологических решений, на который не распространяются рестрикции действующего правового регулирования, получил свое развитие практически во всех странах мира. Российская Федерация не является исключением. В настоящее время принят и вступил в силу Федеральный закон «Об экспериментальных правовых режимах в сфере цифровых инноваций в Российской Федерации» от 31.07.2020 № 258-Ф3, а также ряд подзаконных нормативных правовых актов. Вместе с тем практическая реализация нового правового явления в зарубежных странах опережает теоретическое осмысление стремительно развивающегося института. Более того, в отечественной литературе отсутствуют исследования, посвященные вопросу классификации подходов к регулированию цифровых инноваций и «регулятивных песочниц», различных видов экспериментальных правовых режимов. В настоящей работе изучаются выделяемые в мировой практике виды регулятивных песочниц, а также предлагаются иные классификации экспериментальных правовых режимов.

\section{Подходы к регулированию инноваций}

В зарубежной литературе выделяются четыре подхода к регулированию финтеха [11, c. 7]. Первым подходом является «ждать и наблюдать» (от англ. wait-and-see). При нем регулирующие органы осуществляют мониторинг развития технологий, вмешиваясь в этот процесс лишь в случае необходимости. В качестве примера можно привести равноправное кредитование (Peer-2-Peerlending) и мобильные платежи в Китае. Данное явление появилось в 2007 г., а по состоянию на 2018 г. функционировало уже более 8000 Р2Р платформ, включающих в себя более 50 млн зарегистрированных пользователей, общий оборот которых составил 17,8 млрд китайских юаней. Регулятор вмешался в рынок лишь в 2016 г. [4], когда почти 1/4 существующих платформ испытывала трудности по исполнению своих обязательств перед инвесторами, а почти 40 \% платформ были признаны финансовыми пирамидами [5]. Проиллюстрировать рассматриваемый подход можно также на примере заявления Центрального Банка Ирландии в отношении криптовалют, в котором отмечалось отсутствие необходимости создания специального регулирования новых форм старых видов деятельности, которое в том числе повлекло бы за собой официальное признание неустоявшейся и неоднородной сферы [9].

Второй подход - «пробовать и обучаться» (от англ. test-and-learn). В данном случае регулятор создает индивидуальные условия для каждого конкретного участника, позволяя бизнесу функционировать в ограниченной, но живой среде с определенными исключениями. Такой подход использовался в 2007 г., когда Центральный Банк Кении создал специальные условия для инициативы Safaricom 
в сфере мобильных денежных переводов. В результате созданный сервис M-Реsа стал крупнейшим средством денежных переводов в Кении [3]. Позднее он также был запущен в Танзании, Афганистане, Южной Африке и Индии.

В соответствии с третьим подходом «помощник в инновациях» (от англ. innovation facilitators) - государство выступает катализатором развития инноваций, внедряя центры инноваций, «регулятивные песочницы» или регтех лаборатории. Последние отличаются от «песочниц», поскольку имеют более узкую сферу действия. Регтех (regulatory technology) - это использование финансовыми организациями инновационных технологий для повышения эффективности выполнения регуляторных требований и управления рисками [2, с. 7]. Данный подход становится наиболее распространенным, поскольку позволяет оперативно реагировать на необходимость изменения регулирования в связи с появлением новых технологий. В качестве примера можно привести инновационный хаб Управления денежного обращения Гонконга занимающийся исследованием технологии распределенного реестpa (DLT) для оцифровки процессов торгового финансирования, влияния BigTech на финансовые рынки и применения технологии искусственного интеллекта (AI) в банковской сфере [7].

Четвертый подход, в свою очередь, предполагает создание новых законов или изменение действующего законодательства для его соответствия новым бизнес-моделям инновационных организаций («регулирующиезаконы и реформы», от англ. regulatory laws and reform). По такому пути пошла Мексика, приняв в 2018 г. рамочный закон о финансовых технологиях, направленный на компании, ориентированные на краудфандинг, виртуальные активы и т. д. [6].

Представляется возможным адаптировать наименования данных подходов следующим образом: наблюдательный, апробационный, стимулирующий и реформационный.

Как следует из содержания данных подходов, экспериментальные правовые режимы могут устанавливаться регулирующими органами лишь в рамках использования стимулирующего метода, то есть в ситуации, когда государство сознательно создает условия для развития рынка инновационных технологий и помогает участникам экспериментов преодолеть сложности, вызванные неадаптированным правовым регулированием. Таким образом, подходы к регулированию инноваций являются более широким явлением, чем экспериментальные правовые режимы, а «регулятивные песочницы» выступают лишь в качестве одного из проявлений стимулирующего подхода (наравне с инновационными хабами и регтех лабораториями).

\section{Виды экспериментальных правовых режимов}

В рамках стимулирующего подхода представляется возможным классифицировать регулятивные песочницы по различным основаниям.

По своим целям «песочницы» делят на следующие виды: а) ориентированные на оценку регулятивного воздействия; б) ориентированные на инновации; в) тематические; г) трансграничные $[11$, с. 6]. В первом случае осуществляется мониторинг политики регулирования. Второй вид «песочниц» направлен на снижение стоимости выхода бизнеса на регулируемый рынок, что позволяет организациям проверять рыночную жизнеспособность новых бизнес-моделей. Целью тематических «регулятивных песочниц» является ускорение принятия конкретной политики или нововведений, поддержка развития какого-либо сектора или даже конкретных продуктов, нацеленных на определенные сегменты населения. Трансграничные (или также межюрисдикционные) «песочницы» нацелены на улучшение трансграничной гармонизации регулирования и более быстрое развитие финтех-компаний на международном рынке. Примером такого вида «песочниц» является GFIN - Глобальная сеть финансовых инноваций (The Global Financial Innovation Network), инициированная Управлением по финансовому регулированию и надзору Великобритании, включающая в себя взаимодействие 23 регулирующих органов 16 стран [10]. Отметим, что в данной классификации представляется достаточно спорным основание для выделения видов: целью тематических «песочниц» может также выступать 
ориентация на инновации, а трансграничные режимы могут одновременно являться и тематическими, и ориентированными на оценку регулятивного воздействия.

Трансграничные экспериментальные правовые режимы также можно выделить наравне с национальными и региональными «регулятивными песочницами» по такому основанию, как территория функционирования. Национальные правовые эксперименты охватывают территорию всего государства, а региональные - отдельную географическую зону страны. Так, ч. 2 ст. 5 Федерального закона от 31.07.2020 № 258-Ф3 закрепляет норму о том, что законодательством субъекта Российской Федерации может быть предусмотрено установление экспериментального правового режима в субъекте Российской Федерации, в том числе определен порядок его установления в рамках полномочий органов государственной власти субъектов Российской Федерации по предметам ведения субъектов Российской Федерации и полномочий органов государственной власти субъектов Российской Федерации по предметам совместного ведения Российской Федерации и субъектов Российской Федерации. Между тем единственным фактическим региональным экспериментальным правовым режимом в РФ является внедрение технологий искусственного интеллекта в Москве, установленное Федеральным законом от 24.04.2020 № 123-Ф3 «О проведении эксперимента по установлению специального регулирования в целях создания необходимых условий для разработки и внедрения технологий искусственного интеллекта в субъекте Российской Федерации - городе федерального значения Москве и внесении изменений в ст. 6 и 10 Федерального закона «О персональных данных».

По субъекту проведения экспертизы экспериментальные правовые режимы можно разделить на правовые эксперименты, оценку которых производит постоянный состав экспертов, и правовые эксперименты, оценку которых проводят привлеченные специалисты. В частности, Банком Таиланда был создан специальный финтех департамент, включающий в себя как IT-специалистов, так и специалистов в области регулирования. Помимо этого, Банк Таиланда создает кросс- функциональные команды, состоящие из представителей различных отделов для экспертизы конкретных проектов [8, с. 7].

По продолжительности тестирования регулятивные песочницы могут быть краткосрочными, среднесрочными и долгосрочными. Обычно «регулятивные песочницы» запускают на срок от двух недель до двух лет. Российское законодательство допускает установление экспериментальных правовых режимов на срок до трех лет. В связи с этим предлагается относить к краткосрочным экспериментам правовые режимы, устанавливаемые на срок до одного месяца, к среднесрочным - на срок свыше одного месяца и до одного года, а к долгосрочным - регулятивные песочницы, продолжительность которых превышает один год.

По виду продвигаемых решений экспериментальные правовые режимы делятся на общие и специальные. Часть «песочниц» может быть направлена на какую-либо широкую сферу цифровых инноваций, например искусственный интеллект или финтех в целом. Вместе с тем тестироваться для последующего внедрения могут и специальные узконаправленные технологии (блокчейн, онлайн-платежи и т. д.).

По мерам защиты потребителя «песочницы» могут быть: а) включающими внутренние меры защиты потребителя; б) выполняющими внешние требования регулятора; в) комбинированные. Экспериментальный правовой режим, в котором на этапе моделирования его концепции не продуманы надлежащие меры защиты, несут в себе потенциальные риски для рынка и конечного потребителя. «Песочница» должна предоставлять однозначную информацию о статусе субъекта экспериментального правового режима, а также о собираемых и обрабатываемых данных потребителей. Помимо добросовестности инициатора «песочницы» на этапе ее дизайна, ключевую роль должен, безусловно, играть регулятор, создающий нормативно установленные требования к необходимым мерам защиты потребителей.

Следующие две классификации применимы исключительно к российскому опыту построения экспериментальных правовых режимов и обусловлены национальным законодательством в указанной сфере. 
По регулирующему органу экспериментальные правовые режимы можно разделить на «песочницы», подведомственные Министерству экономического развития РФ [1] и подведомственные Центральному Банку РФ. Указанное деление обусловлено тем, что в соответствии с п. 7 ст. 2 ФЗ «Об экспериментальных правовых режимах в сфере цифровых инноваций в Российской Федерации» от 31.07.2020 № 258-Ф3 регулирующим органом экспериментальных правовых режимов в сфере финансового рынка является Банк России.

Классификация по сфере применения технологий включает в себя экспериментальные правовые режимы в следующих областях: 1) медицинская деятельность; 2) проектирование, производство и эксплуатация транспортных средств, в том числе высокоавтоматизированных транспортных средств и беспилотных воздушных судов, аттестация их операторов, предоставление транспортных и логистических услуг и организация транспортного обслуживания; 3) сельское хозяйство; 4) финансовый рынок; 5) продажа товаров, работ, услуг дистанционным способом; 6) архитектурно-строительное проектирование, строительство, капитальный ремонт, реконструкция, снос объектов капитального строительства, эксплуатация зданий, сооружений; 7) предоставление государственных и муниципальных услуг и осуществление государственного контроля (надзора) и муниципального контроля; 8) промышленное производство (промышленность); 9) иные направления разработки, апробации и внедрения цифровых инноваций, установленные Правительством Российской Федерации. Российское законодательство принципиально отличается от зарубежного опыта, так как не ограничивает правовые эксперименты исключительно финтех сферой, а предполагает возможность апробации технологий и в иных нормативно установленных сферах деятельности.

\section{Выводы}

Проведенное исследование позволяет выделить следующие подходы к регулированию инноваций в мире: наблюдательный, апробационный, стимулирующий и реформаци- онный. В свою очередь, классифицировать «регулятивные песочницы» на основе российского и зарубежного опыта можно по целям: a) ориентированные на оценку регулятивного воздействия; б) ориентированные на инновации; в) тематические; г) трансграничные; по территории функционирования: а) трансграничные; б) национальные; в) региональные; по субъекту проведения экспертизы: а) правовые эксперименты, оценку которых производит постоянный состав экспертов; б) правовые эксперименты, оценку которых проводят привлеченные специалисты; по продолжительности тестирования: а) краткосрочные; б) среднесрочные; в) долгосрочные; по виду продвигаемых решений: a) общие и б) специальные; по мерам защиты потребителя: а) включающие внутренние меры защиты потребителя; б) выполняющие внешние требования регулятора; в) комбинированные. На основе российского законодательства экспериментальные правовые режимы можно разделить в зависимости от регулирующего органа на «песочницы»: a) подведомственные Министерству экономического развития РФ; б) подведомственные Центральному Банку РФ; в зависимости от сферы применения технологий на экспериментальные правовые режимы в следующих областях: а) медицинская деятельность; б) проектирование, производство и эксплуатация транспортных средств, в том числе высокоавтоматизированных транспортных средств и беспилотных воздушных судов, аттестация их операторов, предоставление транспортных и логистических услуг и организация транспортного обслуживания; в) сельское хозяйство; г) финансовый рынок; д) продажа товаров, работ, услуг дистанционным способом; е) архитектурно-строительное проектирование, строительство, капитальный ремонт, реконструкция, снос объектов капитального строительства, эксплуатация зданий, сооружений; ж) предоставление государственных и муниципальных услуг и осуществление государственного контроля (надзора) и муниципального контроля; з) промышленное производство (промышленность); и) иные направления разработки, апробации и внедрения цифровых инноваций, установленные Правительством Российской Федерации. 


\section{ПРИМЕЧАНИЕ}

${ }^{1}$ Публикация подготовлена при поддержке Российского фонда фундаментальных исследований (проект № 20-011-00583 А).

The chapter was prepared with financial support of the Russian Fund for Fundamental Research. Grant of the RFFR 20-011-00583 A.

\section{СПИСОК ЛИТЕРАТУРЫ}

1. Постановление Правительства Российской Федерации от 07.10.2020 № 1618 «О внесении изменения в пункт 1 Положения о Министерстве экономического развития Российской Федерации». Электрон. текстовые дан. - Режим доступа: http:// publication.pravo.gov.ru/Document/View/ 0001202010130016(дата обращения: 09.05.2021).-Загл. с экрана.

2. Центральный Банк Российской Федерации. Основные направления развития финансовых технологий на период 2018-2020 гг. - Электрон. текстовые дан. - Режим доступа: http://www.cbr.ru/ statichtml/file/41186/on_fintex_2017.pdf(дата обращения: 09.05.2021). - Загл. с экрана.

3. Batchelor, S. Changing the Financial Landscape of Africa: An Unusual Story of Evidenceinformed Innovation, Intentional Policy Influence and Private Sector Engagement / S. Batchelor // IDS Bulletin, 2012. - Vol. 43 (5). - P. 84-90. - DOI: https:// doi.org/10.1111/j.1759-5436.2012.00367.x.

4. China Banking Regulatory Commission. Interim Measures on Administration of the Business Activities of Peer-to-Peer Lending Information Intermediaries. - Electronic text data. - Mode of access: https://dfsobservatory.com/content/china-bankingregulatory-commission-interim-measuresadministration-business-activities-peer (date of access: 09.05.2021). - Title from screen.

5. Holmes, C. The Rise and Fall of P2P Lending in China / C. Holmes. - Electronic text data. - Mode of access: https://www.finextra.com/blogposting/17107/ the-rise-and-fall-of-p2p-lending-in-china (date of access: 09.05.2021). - Title from screen.

6. How Regulators Respond To FinTech: Evaluating the Different Approaches. Sandboxes and Beyond // Fintech Note. - No. 4. - Electronic text data. Mode of access: https:/openknowledge.worldbank.org/ handle/10986/33698License: CC BY 3.0 IGO (date of access: 09.05.2021). - Title from screen.

7. Ley para Regular las Instituciones de Tecnologia Financiera. - Electronic text data. - Mode of access: http://www.diputados.gob.mx/LeyesBiblio/ pdf/LRITF_090318.pdf(date of access: 09.05.2021). Title from screen.
8. OpenGovAsia. Hong Kong Working to Foster FinTech Ecosystem. - Electronic text data. - Mode of access: https://opengovasia.com/hong-kong-workingto-foster-fintech-ecosystem/ (date of access: 09.05.2021). - Title from screen.

9. The Alliance for Financial Inclusion. Creating Enabling Fintech Ecosystems: The Role of Regulators. Electronic text data. - Mode of access: https://www.afiglobal.org/sites/default/files/publications/2020-01/ AFI_FinTech_SR_AW_digital_0.pdf (date of access: 09.05.2021). - Title from screen.

10. Tomorrow's Yesterday: Financial Regulation and Technological Change. - Electronic text data. - Mode of access: https://www.centralbank.ie/news/article/ financial-regulation-and-technological-change-gerrycross (date of access: 09.05.2021). - Title from screen.

11. The GFIN Cross-Border Testing. - Electronic text data. - Mode of access: https://www.thegfin.com/ crossborder-testing (date of access: 09.05.2021). - Title from screen.

\section{REFERENCES}

1. Postanovlenie Pravitel'stva Rossijskoj Federacii ot 07.10.2020 № 1618 «O vnesenii izmeneniya $v$ punkt 1 Polozheniya o Ministerstve ekonomicheskogorazvitiya Rossijskoj Federacii» [Resolution of the Government of the Russian Federation Dated 07.10.2020 No. 1618 “On Amending Clause 1 of the Regulation on the Ministry of Economic Development of the Russian Federation"]. URL: http:// publication.pravo.gov.ru/Document/View/ 0001202010130016 (accessed 9 May 2021).

2. Central'nyj Bank Rossijskoj Federacii. Osnovnye napravleniya razvitiya finansovyh tekhnologij na period 2018-2020 gg. [Central Bank of the Russian Federation. The Main Directions of Development of Financial Technologies for the Period 2018-2020]. URL: http://www.cbr.ru/statichtml/file/ 41186/on_fintex_2017.pdf(accessed 9 May 2021).

3. Batchelor S. Changing the Financial Landscape of Africa: An Unusual Story of Evidence-informed Innovation, Intentional Policy Influence and Private Sector Engagement. IDSBulletin, 2012, vol. 43 (5), pp. 84-90.DOI: https://doi.org/10.1111/j.1759-5436.2012.00367.x.

4. China Banking Regulatory Commission. Interim Measures on Administration of the Business Activities of Peer-to-Peer Lending Information Intermediaries. URL: https://ffsobservatory.com/ content/china-banking-regulatory-commissioninterim-measures-administration-business-activitiespeer (accessed 9 May 2021).

5. Holmes, C. The Rise and Fall of P2P Lending in China. URL: https://www.finextra.com/blogposting/ $17107 /$ the-rise-and-fall-of-p2p-lending-in-china (accessed 9 May 2021). 
6. How Regulators Respond To FinTech: Evaluating the Different Approaches. Sandboxes and Beyond. Fintech Note, no. 4. URL: https:// openknowledge.worldbank.org/handle/10986/ 33698License: CC BY 3.0 IGO (accessed 09 May 2021).

7. Ley para Regular las Instituciones de Tecnologia Financiera. URL: http://www.diputados. gob.mx/LeyesBiblio/pdf/LRITF_090318.pdf (accessed 9 May 2021).

8. OpenGovAsia. Hong Kong Working to Foster FinTech Ecosystem. URL: https://opengovasia.com/ hong-kong-working-to-foster-fintech-ecosystem/ (accessed 9 May 2021).
9. The Alliance for Financial Inclusion. Creating Enabling Fintech Ecosystems: The Role of Regulators. URL: https://www.afi-global.org/sites/ default/files/publications/2020-01/AFI_FinTech_SR_ AW_digital_0.pdf(accessed 9 May 2021).

10. Tomorrow's Yesterday: Financial Regulation and Technological Change. URL: https:// www.centralbank.ie/news/article/financial-regulationand-technological-change-gerry-cross (accessed 9 May 2021).

11. The GFIN Cross-Border Testing. URL: https:/ /www.thegfin.com/crossborder-testing (accessed 9 May 2021).

\section{Information About the Author}

Vladislav O. Makarov, Candidate of Sciences (Jurisprudence), Associate Professor, Department of Constitutional and Municipal Law, Volgograd State University, Prosp. Universitetsky, 100, 400062 Volgograd, Russian Federation, makarov.legal@gmail.com, https://orcid.org/0000-0002-3818-6328

\section{Информация об авторе}

Владислав Олегович Макаров, кандидат юридических наук, доцент кафедры конституционного и муниципального права, Волгоградский государственный университет, просп. Университетский, 100, 400062 г. Волгоград, Российская Федерация, makarov.legal@gmail.com, https://orcid.org/0000-0002-3818-6328 